\title{
Energy spectral properties of the twin-CME driven shocks
}

\author{
Xin Wang ${ }^{* \dagger}$ \\ Xinjiang Astronomical Observatory, Chinese Academy of Sciences, Urumqi 830011, China \\ CAS Key Laboratory of Solar Activity, NAOC, Beijing 100012, China \\ Key Laboratory of Radio Astronomy, Chinese Academy of Sciences, Nanjing 210008, China \\ E-mail: wangxinexao.ac.cn
}

Yihua Yan

CAS Key Laboratory of Solar Activity, NAOC, Beijing 100012, China

\section{Mingde Ding}

Key Laboratory of Modern Astronomy and Astrophysics (Nanjing University), Ministry of Education, Nanjing 210093, China

\section{Na Wang \\ Hao Shan \\ Xinjiang Astronomical Observatory, Chinese Academy of Sciences, Urumqi 830011, China}

\begin{abstract}
More and more observations show that large ground-level enhancement (GLE) events are often associated with twin coronal mass ejections (CMEs). In the twin-shock scenario, the preceding CME-driven shock provides an enhanced-level turbulence at the upcoming CME-driven shock. In this work, we focus on the GLE event on 2012 May 17, which would be associated with the twinshock interactions. Using the particle simulation method to examine the effect of the preceding CMEs being surrounded by the forthcoming CMEs on the energy spectrum. We use the preceding CMEs-driven shock to produce an enhanced seed population injecting into the later CME-driven shock for particle re-acceleration. As results, we simulate the twin-shock interactions and obtain the compound proton spectrum. Therefore we suggest that the twin-shock interactions would produce an GLE event with an "ankle-like" proton spectral slope.
\end{abstract}

35th International Cosmic Ray Conference ICRC2017-

10-20 July, 2017

Bexco, Busan, Korea

\footnotetext{
* Speaker.

${ }^{\dagger}$ This work is supported by the Xinjiang Natural Science Foundation No. 2014211A069. Part supported by CAS Key Laboratory of Solar Activity, NAOC, and NSFC (No.11673056).
} 


\section{Introduction}

Interplanetary coronal mass ejections (ICMEs) are one of the two major solar wind structures that have been connected with solar energetic particle (SEP) events in the heliosphere [7]. There is a substantial amount of observational evidence to indicate that CME-driven shocks are the source for the gradual SEPs. For example, there is a linear correlation between the logarithmic of proton peak intensity and the logarithmic of speed of their associated CME [5]. As another empirical association, the peak flux of the SEP is rather correlated with the CME speed than with the X-ray flare peak flux [3].

GLE events are one type of large gradual SEP event. Upon examining all 16 GLEs in solar cycle 23, Li et al. [4] proposed a "twin-CME" scenario for GLE events and large SEP events. In this scenario, two CMEs go off closely in time from the same active region (AR). The first CME drives a shock which generates a very turbulent downstream. The first shock can also pre-accelerate particles, although not necessarily to very high energies.

The "twin CME" scenario focusing on the presence of two CME-driven shocks, resembles that of sympathetic eruptions. Because the "twin CME" scenario suggests that efficient acceleration occurs at the shock driven by the second CME, it implies that a single CMEs may not lead to large SEP events. Wang et al. $[12,13]$ examine an isolated CME-driven shock can accelerate the proton to the largest attainable energies of SEP events up to $\sim 5 \mathrm{MeV}$. Ding et al. [2] tested the "twin CME" scenario against all large SEP events and western CMEs with speed $>900 \mathrm{kms}^{-1}$ in solar cycle 23 and found that most of the large SEP events agree with the "twin CME" scenario and many single fast CMEs do not lead to large SEP events. Wang et al. [14] investigate the interactions between the CME-driven shock and Earth's bow shock, which can produce the highest attainable proton energy reaching to $\sim 20 \mathrm{MeV}$, but with a soft energy spectral slope beyond the "break" point at $\sim$ $5.5 \mathrm{MeV}$. Here, we propose a simulation of the twin-shock interactions for further investigating a high energy "tail" and the energy spectral properties.

According to the report of the NOAA, the 2012 May 17 GLE events are originated from a long-duration M5.1 X-ray flare from 01:25 to 02:14 UT. The source of the solar active region (AR) is 11476 and its location at the area (N11, W86). As far as the level of M5.1 is concerned, the flare was not particularly large. But all three energy channels of the GOES spacecraft showed the prompt rises, indicating enough efficient particle acceleration processes. We suggest that there were two eruptions and two CMEs in the 2012 May 17 event. Both CMEs were fast and were clearly seen from STEREO-B coronagraph observation. The observed ground-level enhancement of energetic particles in the 2012 May 17 GLE event and the fact that there were two fast CMEs in the event supports the recently proposed "twin CME" scenario for GLE events.

\section{Model}

This model describes the "twin-shock" interactions on 2012 May 17 GLE event at the interplanetary space less than $5 R_{\odot}$ (here, $R_{\odot}$ is the radii of the Sun). Since the AR is located at W88 from the Earth, the event was a backside event for STEREO-B. The propagation directions of the two CMEs are also shown in coronagraph observations made by SOHO/LASCO. While the two CMEs were clearly seen from STEREO-B and SOHO, they overlapped and appeared to be propagating 
together and were hardly distinguishable from the STEREO-A. Shen et al. [8] use the Graduated Cylindrical Shell (GCS) model to give the height $h$ of the two CMEs at different times and the parameters of the longitude $\phi$, the latitude $\theta$, and others. The differences of $\delta \theta$ and $\delta \phi$ between the two CMEs are $11^{\circ}$ and $31^{\circ}$. This means that there are two distinct CMEs in this event and they have different but close propagation directions. According to the calculated list of the hight of the two-CMEs at different times, they gave the hight $h_{1}=4.5 R_{\odot}$ for CME1 and the hight $h_{2}=4.82 R_{\odot}$ for CME2 at the time of 02:00UT. The difference of the hights of the two CMEs is $0.32 R_{\odot}$. They also used the relationship between the hight of CMEs and the propagation time [9] to obtain the speeds of the two CMEs, which are $1258 \pm 352 \mathrm{kms}^{-1}$ and $1539 \pm 352 \mathrm{kms}^{-1}$ for CME1 and CME2, respectively.

Fig. 1 shows a schematic diagram of the twin-shock model. The left reflective wall represents CMEs and produce the shock No.1 and No.2 propagating from the left of the simulation box to the right. After a duration, the shock No.1 propagating into the center of the simulation box, the second CME appears at the right reflective wall and produces the shock No.2. The shock No.2 also propagates into the simulation box with a relative bulk speed $\Delta U_{2}$ to the shock No.1. The two shocks are indicated by the two blue vertical bars at the simulation box in Fig. 1. Precedently, the upstream bulk flow speed of the shock No.1 is $\Delta U_{1}$ and the downstream bulk flow speed is zero at the rest reference frame of the shock No.1. When the shock No.2 appears at the left boundary, then there is an interaction with the shock No.1 with a relative bulk flow speed $\Delta U_{2}$. So at the rest reference frame of the shock No.2, both of the upstream and downstream bulk speeds of the shock No.1 add an increment of speed $\Delta U_{2}$ become to be the $\Delta U_{1}+\Delta U_{2}$ and $\Delta U_{2}$, respectively. Correspondingly, the upstream and downstream bulk speeds of the shock No.2 become the $\Delta U_{2}$ and zero. In the twin-shock scenarios, since there is a relative bulk speed $\Delta U_{2}$ between the two shocks, the downstream bulk flow of the shock No.1 is compressed by the shock No.2 while the two CMEs commencing a collision. So the heating and accelerated ions at the downstream of the shock No.1 can be re-acceleration by Fermi acceleration processes at the shock No.2. We perform a Mote-carlo code to simulate the twin-shock scenario in detail for investigating the alteration of the energy spectrum undergoing the "twin-CMEs" collisions.

In this Monte Carlo method, we apply an initial number density of particles $n_{0}$ in the upstream bulk flow of the shock No.1, which obeys a Maxwellian distribution with a thermal speed $v_{\text {th }}$. Initially, the upstream bulk flow of the shock No.1 remains the bulk speed with $\Delta U_{1}$ until there is a collision between the CME1 and the CME2 at the left boundary of the simulation box. Here, we set the total simulation time is $t_{\max }$. At the half of the total simulation time, we set the two CMEs begin a collision at the left reflective wall of the simulation box. Then, during the next half of the total simulation time, the particles will undergo re-acceleration processes on the collided region between the twin-shock. We suggest the CME1 would produce the shock No.1 forming a normal power-law energy spectrum, but the CME2 would produce the shock No.2 enhancing the existing normal power-law energy spectrum. In this "twin-shock" scenarios, the twin-CME shocks have a collision with the same direction from the source to the collided location. Therefore the shock No.2 produce a positive effect on the shock No.1 resulting an enhancement for the existing energy spectrum. We predict there would appear a "concave" energy spectrum at a certain energy range, which would be identified by performing the "twin-shock" simulation on the 2012 May 17 SEP event. 


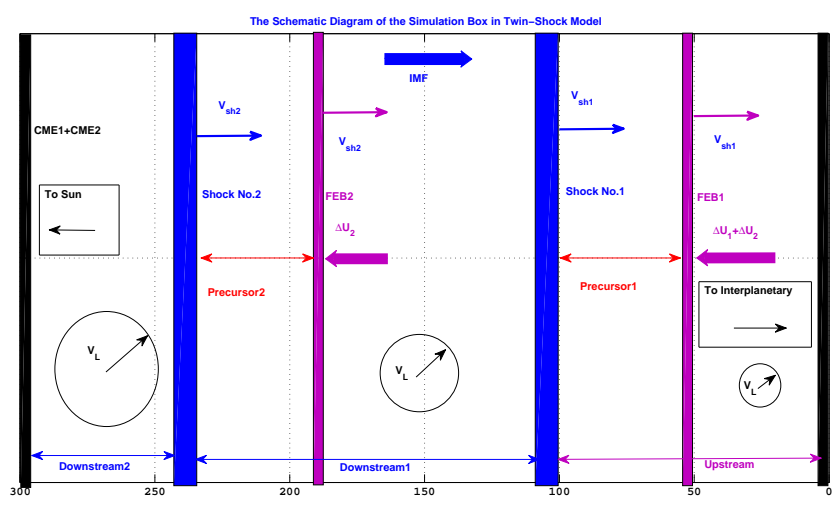

Figure 1: A schematic diagram of the twin-shock simulation box. The left reflective wall represents the CMEs produce shocks No.1 and No.2 (representing by two blue vertical bars ) propagating from the left boundary to the right of the simulation box. After a duration, the CME1 produce shock No.1 propagating into the center of the simulation box, the CME2 produces shock No.2 appearing at the left boundary of the simulation box. Then the twin shocks evolve into the simulation box with an interaction.

Monte Carlo method applies a scattering law for particle diffusive processes on shocked plasmas. We assume that the particles scatter elastically off the background scattering centers with their scattering angles obeying an isotropic distribution in their local frame. In this scattering scenario, the assumption of elastic scattering requires that scattering centers are frozen into the background fluid [1].

All the simulated parameters are listed in Table 1 . According to the observation, we adjust the observed parameters to apply the simulation. We present the scaled values of the parameters as follows. The relative upstream bulk flow speed of the shock No.1 is $800 \mathrm{kms}^{-1}$, the relative upstream bulk flow speed of the shock No.2 is $400 \mathrm{kms}^{-1}$. The initial thermal velocity $v_{L}$ is $26.9 \mathrm{kms}^{-1}$. The box size is set to be the $5 R_{\odot}$ (where $R_{\odot}$ is the radii of the Sun). The total time of the simulation is 343 minutes. The scattering time $\tau_{0}$ and the time step $d t$ can be referenced to Knerr et al. [6].

The above scaled values of the parameters are corresponded to the follow dimensionless parameters: upstream bulk speeds $\Delta U_{1}=0.6$ and $\Delta U_{2}=0.3$, initial local thermal velocity $v_{\mathrm{L}}=0.02$, the total size of the box $X_{\max }=300$, the total simulation time $t_{\max }=2400$, the constant of the scattering time $\tau_{0}=25 / 30$, and the time step $d t=\tau_{0} / 12.5$. Those above dimensionless values can be scaled by distance $(X)$, time $(t)$, and velocity $(U)$ scaling factors: $X_{\text {scale }}=5 R_{\odot} / 300, U_{\text {scale }}=800 \mathrm{kms}^{-1} / 0.6$, and $t_{\text {scale }}=X_{\text {scale }} / U_{\text {scale }}$, respectively. In addition, we give the simulation box grids of $m_{\mathrm{x}}=600$, and the initial density of particles in each grid is $n_{0}=300$. The total simulation time is divided into ten time durations represented by sequences $\mathrm{Q}=1,2,3, \ldots$ and 10 .

\section{Results}

\subsection{Shock Evolution}

Fig. 2 shows the velocity snapshots of the bulk flow with the grids at the simulation times of $\mathrm{t}=240,480,720, \ldots$, and 2400 . In the Fig.2, the bulk flow with upstream velocity $\Delta U_{1}$ of the shock No. 1 before the time $t=1200$ shows a single shock. After the duration of the half of the total 
Table 1: The Simulation Parameters

\begin{tabular}{|l|c|c|}
\hline Physical Parameters & Dimensionless Values & Scaled Values \\
\hline Relative upstream bulk speed 1 & $\Delta U_{1}=0.6$ & $800 \mathrm{kms}^{-1}$ \\
Relative upstream bulk speed 2 & $\Delta U_{2}=0.3$ & $400 \mathrm{kms}^{-1}$ \\
Initial thermal velocity & $v_{\mathrm{L}}=0.02$ & $26.9 \mathrm{kms}^{-1}$ \\
Box size & $X_{\max }=300$ & $5 R_{\odot}$ \\
Total time & $t_{\max }=2400$ & 343 munites \\
Number of zones & $m_{\mathrm{x}}=600$ & $\ldots$ \\
Initial particles per cell & $n_{0}=300$ & $\ldots$ \\
Time durations & $\mathrm{Q}=1,2,3 \ldots 10$ & $\ldots$ \\
\hline
\end{tabular}

Notes: The $R_{\odot}$ is the radii of the Sun. The scale factors for distance, velocity, and time are $X_{\text {scale }}=5 R_{\odot} / 300$, $U_{\text {scale }}=800 \mathrm{kms}^{-1} / 0.6$, and $t_{\text {scale }}=X_{\text {scale }} / U_{\text {scale }}$. The dimensionless values and the scaled values can be transformed by the scaled factors each other.
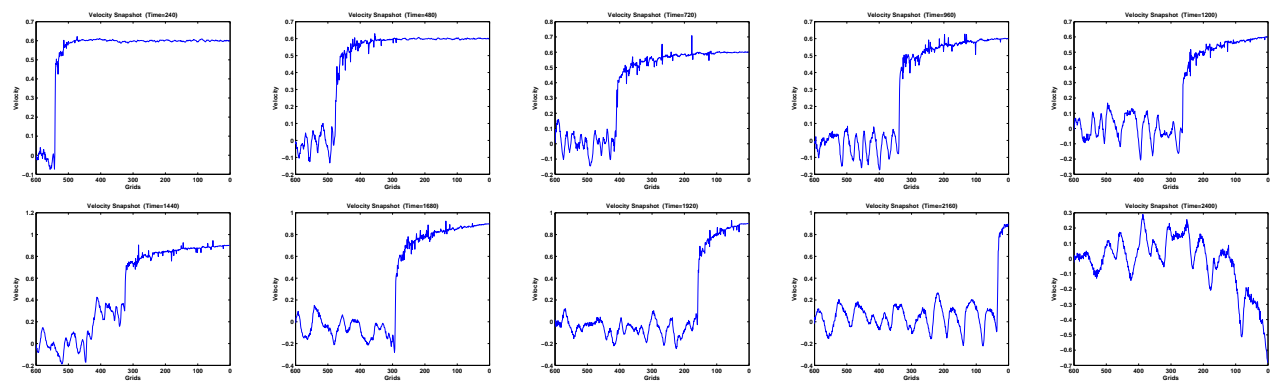

Figure 2: The velocity snapshots of the bulk flow with the grids at a series of the simulation time $\mathrm{t}=240$, $480,720, \ldots$, and 2400 .

simulation time, the bulk flow shows the twin-shock in the simulation box. The upstream bulk speeds of the shock No.1 and shock No.2 become the $\Delta U_{1}+\Delta U_{2}$ and $\Delta U_{2}$ at the new rest reference frame. With the time increasing, shock No.2 compresses the downstream flow of the shock No.1. Then, the bulk-flow speed of the downstream of the shock No.2 becomes zero.
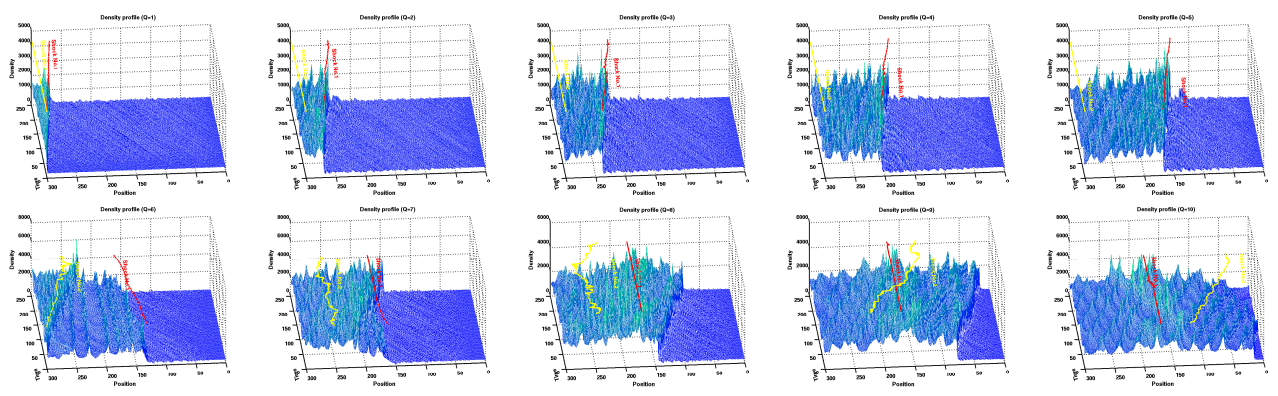

Figure 3: The density profiles of the bulk flow at the durations of the simulation time $Q=1,2,3, \ldots, 10$.

Fig. 3 shows a group of bulk flow density profiles at the durations of $Q=1,2,3, \ldots$, and 10 . The red solid line in each plot represents the position of the shock No.1, respectively. The yellow solid line in each plot represents the position of the shock No.2, respectively. The upper panel shows a series of bulk flow density profiles of the shock No.1 in terms of position with time. The lower 
panel shows a series of bulk flow density profiles of both the shock No.2 and shock No.1 evolving into the simulation box. The upstream bulk flow density of the shock No.1 is the initial density $n_{0}$, and the density of the downstream bulk flow of shock No.1 become about 5 times of the initial upstream density. Once the simulation time has passed the half of the total simulation time $t_{\max }$, the shock No.2 begins to evolve into the simulation system. In the twin-shock scenarios, the shock No. 2 compresses the downstream bulk flow of the shock No.1 resulting an enhancement of the density of the downstream of the shock No.2 with about 8 times of the initial density. High density and high-random velocity of the bulk flow between the twin-shock would amplify the magnetic field for enhancing the particle acceleration efficient. This positive effect of the twin-shock on the particle acceleration would change the existing energy spectral shape.

\subsection{Particle Acceleration}
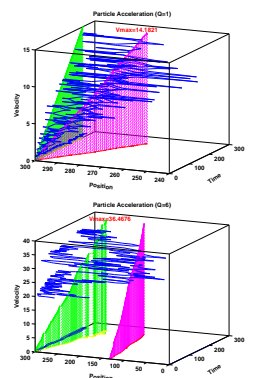
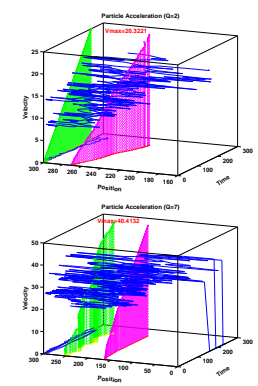
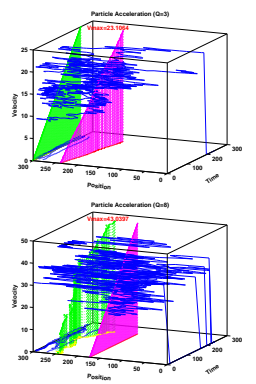
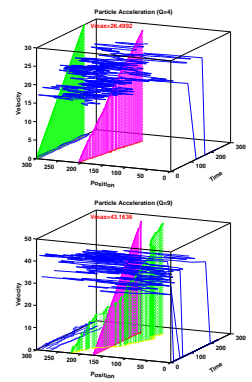
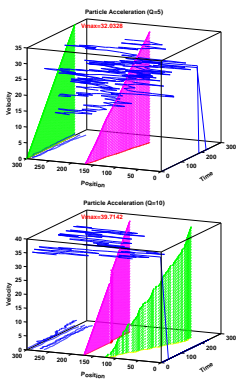

Figure 4: Particle accelerations in the simulation durations $\mathrm{Q}=1,2,3, \ldots$, and 10 . The blue curves in each plot represent particle trajectories. Two shadowed triangles represent the shock No.1 and shock No.2, respectively.

Fig. 4 shows a group of plots for particle acceleration processes at the different durations of $\mathrm{Q}=1,2,3, \ldots$, and 10. A few particles extracted from the simulation box are represented by blue curves in each plot with corresponding index of the time sequence Q. Two triangle shadows representing two shocks in each plot indicate there are two-shock evolutions in the simulation box. The pink triangle shadow represents the shock No.1, and the green triangle shadow represents the shock No.2. At the first half the total simulation time $t_{\max }$, there is only shock No.1 evolving into the simulation box and forms the normal shock structure. Once the simulation time passes the half of the total simulation time $t_{\max }$, the shock No.2 enters into the simulation box for forming twin-shock scenarios. The pre-accelerated particles are accelerated by the shock No.1 in the first half of the total simulation time will re-accelerate by the shock No.2. in the next half of the total simulation time. Then the particle accelerations show an enforcement of the energy gains between the twin shocks. This positive effect of the twin-shock would modify the particle energy spectrum. The lower panel show the twin-shock interaction amplify the magnetic field and produce the higher maximum particle energies than single shock No.1 in the upper panel. In each plot, some of the blue curves show particle accelerations by crossing shock front back and forth with energy gains. Another curves that lie down in the bottom of each plot show particles without acceleration in the downstream regions. Each maximum particle velocity $V_{\max }$ produced in each duration of the simulation is denoted by its value in the corresponding plot. These maximum velocities show that they produce the positive effect of the twin- shock at the next half of the total simulation time. The 
merged precursor region could provide an amplified magnetic field to produce more energetic particles population than those produced in a single shock model, which just produce a regular single power-law spectrum. Actually, the compressed interaction region between twin shocks could lead to a change of the energy spectral slope by the magnified magnetic turbulence.

\subsection{Energy Spectra}

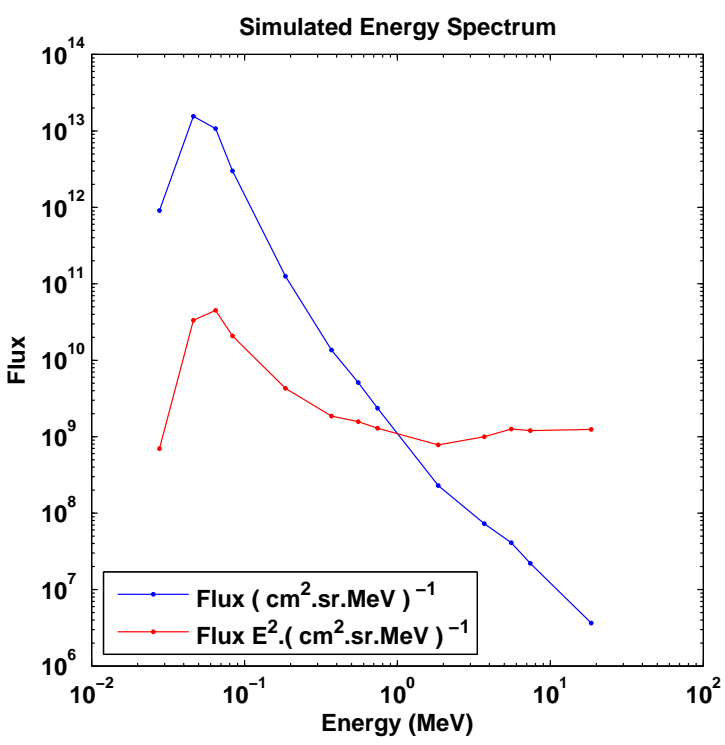

Figure 5: The simulated energy spectrum calculated from the twin-shock scenario. The blue curve represents the particle fluxes with the normal representation, the red curve represents the energy spectrum with a specific representation by using a factor of the square of the energy times the flux.

Fig. 5 shows the simulated energy spectrum with two different representations for particle fluxes. The blue curve represents the particle fluxes with the normal representation, the red curve represents the energy spectrum with a specific representation by using a factor of the square of the energy times the flux. From the Fig .5 , the energy spectrum covers the total energy range from $30 \mathrm{keV}$ to $20 \mathrm{MeV}$. There is a maxwellian thermal peak at $\sim 40 \mathrm{keV}$. Then the suprethermal particle "tail" is shown at the energy range from the $\sim 70 \mathrm{keV}$ to $400 \mathrm{keV}$. At the energy range from the $400 \mathrm{keV}$ to $\sim 2 \mathrm{MeV}$, the energy spectrum shows a softer power-law property with an index more than 2. Comparably, at the energy range from the $\sim 2 \mathrm{MeV}$ to $\sim 20 \mathrm{MeV}$, the energy spectrum shows a harder power-law property with an index less than 2 . The red curve can clearly show that "concave" property in the total energy spectrum. According to our calculation from the red curve, we predict that there would be the "concave" point at $\sim 2 \mathrm{MeV}$. We suggest this "concave" point would occur by means of a positive effect on the pre-acceleration particles in twin-shock interactions.

\section{Summaries and Conclusions}

In summary, we simulate the twin-shock system for predicting the proton spectrum directly. We obtain the total energy spectrum covering the energy range from $30 \mathrm{keV}$ to $20 \mathrm{MeV}$. We also 
find the simulated energy spectrum exhibits the energy spectral "concave" at the energy point of $\sim 2 \mathrm{MeV}$. Comparably, we have ever investigated an energy spectrum "break" at $\sim 5.5 \mathrm{MeV}$ in our previous converging double-shock model. And in another study, we have also predicted the single shock would produce an saturation of the maximum particle energy up to $\sim 5.5 \mathrm{MeV}$. So, why do the converging shocks in previous study would produce a "broken" energy spectrum and the "pileup" twin-shock in present study would produce a "concave" energy spectrum? There would be some reasons: (i) According to the diffusive shock acceleration theory, the acceleration efficient is determined by the diffusive coefficient[10]. The attainable highest energy particle is depended on the diffusive length of particles scaled by the size of the precursor region[11]. At the converging shocks, the precursor region size will be shorten and fewer and fewer high energy particles gain energies resulting a softer energy at the high energy "tail"[14]. (ii) But in the twinshock scenario, the twin-shock interaction can extend the precursor region size and enhance the existing accelerated particle distribution. The pre-accelerated particles caused by the first shock, penetrate into the second shock for re-accelerating and modifying the existing power-law energy spectrum to become a harder energy spectrum. These processes can lead to a"concave" shape on the energy spectrum at a certain energy range. (iii) Furthermore, whatever in the converging shocks and the twin shocks model, both the diffusive particles can extend the energy spectrum up to a few decades of $\mathrm{MeV}$ in their high energy "tails". The specific energy spectral shape would be depended on the type of the collision, in which the important parameter of the precursor size will be decided.

\section{References}

[1] D. C. Ellsion, \& G. P. Double, 2004, Astropart. Phys., 22, 323

[2] L. G. Ding, Y. Jiang, L. Zhao, \& G. Li, 2013, ApJ, 763, 30

[3] N. Gopalswamy, S. Yashiro, G. Michäk, et al. 2002, ApJL, 572, L103

[4] G. Li, R. Moore, R. A. Mewaldt, L. Zhao, \& A. W. Labrador, 2012, Space Sci. Rev. , 171, 141

[5] S. W. Kahler, \& A. Vourlidas, 2013, ApJ, 769, 143

[6] J. M. Knerr, J. R. Jokipii, \& D. C. Ellison, 1996, ApJ, 458, 641-652

[7] D. V. Reames, 1999, Space Sci. Rev. , 90, 413

[8] C. Shen, G. Li, X. Kong, J. Hu, X. D. Sun, et al. 2013, ApJ, 763,114

[9] A. F. R. Thernisien, A. Vourlidas, \& R. A. Howard, 2009, SoPh, 256, 111-130

[10] X. Wang, N. Wang, \& Y. H. Yan, 2013, ApJS, 209,18

[11] X. Wang, \& Y. H. Yan, 2011, Astron. Astrophys., 530, A92

[12] X. Wang, \& Y. H. Yan, 2012, Res. Astron. Astrophys., 12, 1535-1548

[13] X. Wang, Y. H. Yan, M. D. Ding, N. Wang, \& H. Shan, 2016, Res. Astron. Astrophys., 16, 32

[14] X. Wang, J. Giacalone, Y. H. Yan, M. D. Ding, N. Wang, \& H. Shan,2017, ApJ, 842, 74 